\title{
Localización de locales comerciales: un enfoque de selección multicriterio
}

DOI: https://doi.org/10.21158/01208160.n87.2019.2446

Fecha de recepción: 29 de enero de 2019

Fecha de aprobación: 20 de diciembre de 2019
Adel Mendoza-Mendoza

Universidad del Atlántico adelmendoza@mail.uniatlantico.edu.co

Enrique Delahoz-Dominguez Universidad Tecnológica de Bolívar edelahoz@utb.edu.co

Daniel Mendoza-Casseres Universidad del Atlántico danielmendoza@mail.uniatlantico.edu.co

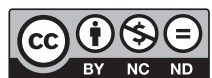

Cómo citar este artículo / To reference this article / Comment citer cet article / Para citar este artigo:

Mendoza-Mendoza, A.; Delahoz-Dominguez, E.; Mendoza-Casseres, D. (2019). Localización de locales comerciales: un enfoque de selección multicriterio. Revista Escuela de Administración de Negocios, (87), 207-219. DOI: https://doi.org/10.21158/01208160. $\mathrm{n} 87.2019 .2446$

\section{Resumen}

La decisión referente a la localización de un local comercial es de carácter estratégico, ya que se deben estudiar las diferentes alternativas de acuerdo con múltiples factores, de manera que puede considerarse un proceso complejo de toma de decisiones multicriterio o MCDM. En el presente trabajo se propone una metodología para la selección de localización de locales comerciales que integra el proceso de jerarquía analítica difuso o AHP difuso, el cual se utiliza a fin de ponderar los criterios para la selección bajo la técnica de orden de preferencia por similitud con la solución ideal o TOPSIS, a fin de seleccionar la mejor opción de localización según las calificaciones obtenidas por todas las alternativas. Se presenta un estudio de caso, con el propósito de ilustrar la aplicabilidad de la metodología propuesta, en el que se tienen cinco centros comerciales como elecciones de localización, en conformidad con cuatro criterios: infraestructura, costo de arrendamiento, accesibilidad de clientes y potencial comercial. Las ponderaciones que alcanzaron cada criterio fueron $11,2 \%, 21,0 \%, 14,3 \%$ y 53,5 \%, respectivamente. Finalmente, no solo se demuestra la aplicabilidad de la metodología para el caso sino los alcances que la misma puede llegar a tener en otros sectores de negocio, pues se presenta como una manera confiable y efectiva para seleccionar la mejor opción entre un grupo de alternativas dependiendo de los diferentes criterios clave.

Palabras clave: selección de locales comerciales; selección multicriterio; local comercial; espacio comercial; infraestructura comercial; potencial comercial.

\footnotetext{
${ }^{1}$ Ingeniero químico- Universidad del Atlántico. Especialista en gerencia de producción y operaciones - Universidad Autónoma del Caribe. Magíster en ingeniería industrial - Universidad del Norte. ORCID: https://orcid.org/0000-0002-4278-1226

${ }^{2}$ Ingeniero Industrial de la Universidad Libre. Especialista en Logística Empresarial de la Universidad del Norte. Máster en Investigación de Operaciones por la Universitat de Barcelona. ORCID: https://orcid.org/0000-0003-2531-6389

${ }^{3}$ Magíster en Ingeniería Industrial de la Universidad de Los Andes. Ingeniero Químico de la Universidad del Atlántico de Barranquilla. ORCID: http://orcid.org/0000-0001-5514-750X
} 


\section{The location of commercial premises: a multi-criteria selection approach}

\section{Abstract}

The decision regarding the location of commercial premises is one of strategic nature, as the different alternatives that may be available must be studied according to multiple factors, that way, it can be considered a complex Multi-Criteria Decision-Making (MCDM) process. This document is intended to propose a methodology for the selection of location of commercial premises that integrates the fuzzy analytical hierarchy process or fuzzy AHP, which is used to weight the selection criteria under the Technique for Order of Preference by Similarity to Ideal Solution (TOPSIS), in order to select the best option for the location according to the qualifications that are obtained from all the alternatives. A case study is presented, with the purpose of illustrating the applicability of the methodology that has been proposed, in which five shopping centers are used as location choices, according to four criteria: infrastructure, cost of renting, accessibility to customers, and commercial potential. The weights that were achieved by each criterion were $11.2 \%, 21.0 \%$, $14.3 \%$, and $53.5 \%$, respectively. Finally, it not only demonstrates the applicability of the methodology for the case, but also, the scope that it can have in other business sectors, since it is presented as a reliable and effective way to select the best option among a group of alternatives, depending on the different key criteria.

Keywords: selection of commercial premises; multi-criteria selection; commercial space; commercial infrastructure; commercial potential.

\section{Localização de instalações comerciais: uma abordagem de seleção multicritério}

\section{Resumo}

A decisão sobre a localização de um local comercial é estratégica, uma vez que as diferentes alternativas devem ser estudadas de acordo com múltiplos fatores, para que possa ser considerado um processo complexo de tomada de decisão por múltiplos critérios ou MCDM. Este artigo propõe uma metodologia para a seleção de locais de instalações comerciais que integra o processo de hierarquia analítica difusa ou AHP difusa, que é usada para ponderar os critérios de seleção na técnica de ordem de preferência por similaridade com a solução ideal ou TOPSIS, para selecionar a melhor opção de localização de acordo com as classificações obtidas por todas as alternativas. Se apresenta um estudo de caso, com o objetivo de ilustrar a aplicabilidade da metodologia proposta, na qual existem cinco shopping centers como opções de localização, de acordo com quatro critérios: infraestrutura, custo do aluguel, acessibilidade do cliente e potencial comercial. Os pesos que atingiram cada critério foram $11,2 \%, 21,0 \%, 14,3 \%$ e 53,5 \%, respectivamente. Finalmente, não apenas é demonstrada a aplicabilidade da metodologia para o caso, mas também o escopo que ela pode ter em outros setores de negócios, uma vez que se apresenta como uma maneira confiável e eficaz de selecionar a melhor opção dentre um grupo de alternativas, dependendo dos diferentes critérios-chave.

Palavras-chave: seleção de instalações comerciais; seleção multicritério; local comercial; espaço comercial; infraestrutura comercial; potencial comercial. 


\section{Localisation des locaux commerciaux: une approche de sélection multicritères}

\section{Résumé}

La décision du choix de l'emplacement d'un local commercial est stratégique; les différentes alternatives doivent être étudiées en fonction de multiples facteurs pour être considérées comme un processus décisionnel multicritères complexe. Cet article propose une méthodologie pour la sélection de l'emplacement des locaux commerciaux qui intègre un processus de hiérarchie analytique diffuse utilisé pour analyser les critères de sélection selon une technique d'ordre de préférence par similitude avec solution idéale ou TOPSIS, afin de sélectionner la meilleure option de localisation en fonction des critères obtenus selon les alternatives choisies. Nous présenterons une étude de cas dans le but d'illustrer l'applicabilité de la méthodologie proposée entre cinq centres commerciaux selon quatre critères de choix d'emplacement: infrastructure, coût de la location, accessibilité clientèle et potentiel commercial. Les pondérations correspondant à chaque critère sont respectivement de 11,2\%, 21,0\%, 14,3\% et 53,5\%. L'applicabilité de la méthodologie est démontrée dans ce cas mais aussi la portée que cette dernière peut avoir dans d'autres secteurs d'activité, car il s'agit d'un moyen fiable et efficace de sélectionner la meilleure option dans un groupe d'alternatives en fonction de différents critères clés de sélection.

Mots-clés: sélection de locaux commerciaux; sélection multicritères; local commercial; espace commercial; infrastructure commerciale; potentiel commercial 


\section{Introducción}

$\mathrm{E}$ proceso de localización de locales comerciales algunas veces se lleva a cabo de manera intuitiva. Este aspecto se debe valorar de la mejor manera posible, ya que es un factor que puede generar ventajas competitivas (Hualpa-Zúñiga y Suárez-Roldán, 2013). Además, difícilmente esta decisión se puede modificar en el corto plazo. La elección de una buena localización juega un papel crucial en las industrias minorista y de servicios, ya que es importante para atraer a más clientes $\mathrm{e}$ impactar, de manera sustancial, en la participación de mercado (Chen y Tsai, 2016). Por esta razón se puede afirmar que se trata de un problema de decisión estratégicamente importante.

Yıldız y Tüysüz (2018), por su parte, señalan cuatro teorías que se desarrollaron con relación a la selección de la localización de locales minoristas: la teoría de lugar central, la teoría de interacción espacial, la teoría de renta de oferta y el principio de diferenciación mínima, así como otras técnicas disponibles. Sevtsuk (2014) menciona que existen modelos matemáticos que permiten predecir ubicaciones potenciales de tiendas minoristas, sin embargo, este problema de localización puede evaluarse según varios criterios. En razón a lo anterior, el objetivo de este trabajo es desarrollar una metodología basada en técnicas de decisión multicriterio para la selección de la localización de tiendas minoristas mediante la combinación entre proceso de jerarquía analítica AHP difuso o FAHP, por sus siglas en inglés, y la técnica para el orden de preferencia por similitud a la solución ideal o TOPSIS, por sus iniciales en inglés. Estas herramientas, en razón a su lógica y estructura metodológica, facilitan este proceso de toma de decisiones empresariales. Los métodos de decisión multicriterio ayudan a los responsables de la toma de decisiones a resolver problemas de decisión complejos que involucran criterios contradictorios, los cuales son difícilmente cuantificables de una manera sistemática y consistente (Sennaroglu y Celebi, 2018).

El artículo se desarrolla de la siguiente manera. En la segunda sección se presentan las técnicas de decisión multicriterio y sus aplicaciones. La metodología propuesta se desarrolla en la tercera sección y, a continuación, en la cuarta sección se presenta un estudio de caso aplicado para la selección de localización de una tienda minorista en Barranquilla. En la quinta sección se analizan los resultados y se finaliza con la presentación de las conclusiones.

\section{Revisión de la literatura}

$\mathrm{E}$ proceso de jerarquía analítica o AHP, por sus siglas en inglés, desarrollado por Saaty (1980), ha sido extensamente utilizado en la toma de decisiones multicriterio. Este método emplea datos cuantitativos y cualitativos, los cuales se traducen en números. El AHP es una teoría de la medición mediante comparaciones por pares. El método se usa con el fin de comparar alternativas y determinar su importancia entre sí, mediante un proceso sistemático y jerárquico (Bhushan y
Rai, 2007; Saaty, 2008). Las comparaciones se realizan mediante una escala de juicios absolutos que representa la medida de dominación de un elemento sobre otro con respecto a un atributo dado. Lidiar con juicios inciertos a fin de expresar la importancia de los criterios indica la posibilidad de usar conjuntos difusos o números borrosos, lo que incorpora la vaguedad del pensamiento humano. Dado que es difícil mediante preferencias cualitativas realizar las estimaciones, se asociará un 
grado de incertidumbre con algunos o todos los pares de valores de comparación en un problema de AHP. Con el fin de incorporar la falta de claridad en la toma de decisiones de múltiples criterios muchos autores han utilizado el proceso de jerarquía analítica difuso o FAHP, tal como lo indican Carlsson y Fullér (1996). En el método FAHP se reemplaza la escala tradicional por una escala numérica triangular difusa, con lo cual se logra un manejo más eficaz de la imprecisión de los juicios lingüísticos de los responsables de la toma de decisiones, y se recibe así una mejor aceptación por parte de los investigadores con relación a la del método original (Santis, Golliat y Aguiar, 2017).

El proceso de jerarquía analítica o AHP es una de las herramientas de análisis multicriterio ampliamente utilizadas en la toma de decisiones para modelar problemas no estructurados en diferentes áreas, como, por ejemplo, en ciencias políticas, económicas, sociales y de gestión. Muchos estudios han aplicado AHP y FAHP en diferentes situaciones y problemas. Así, por ejemplo, el de Dožić, Lutovac y Kalić (2018) en la selección del tipo de aviones para pasajeros; el de Jayawickrama, Kulatunga y Mathavan (2017) con el fin de evaluar la sostenibilidad de una planta de fabricación; el de Gürcan, Beyca, Arslan y Eldemir (2016) en la selección de proveedores; o el de Mosadeghi, Warnken, Tomlinson y Mirfenderesk (2015) que compara los métodos AHP y FAHP en la definición de la extensión de las zonas de uso de la tierra en un escenario de planificación urbana a gran escala. En aplicaciones de localización se destaca el trabajo de Asakereh, Soleymani y Sheikhdavoodi (2017) para ubicación de granjas solares; el de Chauhan y Singh (2016) en la localización de depósitos para residuos sanitarios; y el de Hong y Xiaohua (2011) en la localización de centros logísticos de emergencia.
La técnica para el orden de preferencia por similitud a la solución ideal o TOPSIS, desarrollado por Hwang y Yoon en 1981, es un método simple de clasificación en la concepción y la aplicación. El método estándar TOPSIS intenta elegir alternativas que presenten, de forma simultánea, la distancia más corta desde la solución ideal positiva y la distancia más alejada de la solución ideal negativa. La solución ideal positiva maximiza los criterios de beneficio y minimiza los criterios de costo, mientras que la solución ideal negativa maximiza los criterios de costo y minimiza los criterios de beneficio (Behzadian, Otaghsara, Yazdani e Ignatius, 2012). Al igual que el AHP, la técnica TOPSIS se ha utilizado para diferentes aplicaciones: Jumaah et al. (2018) la utilizaron en la evaluación de los modos de operación de sistemas de posicionamiento global, Walczak y Rutkowska (2017) en la clasificación de proyectos en un presupuesto participativo, y Senouci, Mushtaq, Hoceini y Mellouk (2016) en la selección de interfaces de redes de comunicaciones móviles inalámbricas.

En la aplicación combinada de AHP-TOPSIS se pueden mencionar los trabajos de Hsieh et al. (2018) dirigidos a identificar factores de error humanos en departamentos de emergencia; los de Zhao et al. (2018) para la selección de la ubicación de los centros de distribución; los de Kazazi, Akbari, Jabbari y Asefi (2018) a fin de determinar la ubicación de estacionamientos en consideración a múltiples criterios económicos, sociales y ambientales; los de Alizadeh, Rad y Bazzazi (2016) para seleccionar un método de procesamiento de alúmina; y los de Beikkhakhian, Javanmardi, Karbasian y Khayambashi (2015) utilizados en la evaluación de proveedores. 


\section{Metodología}

T a metodología utilizada en este artículo para la es una combinación de FAHP orientada a definir la ponderación de la jerarquía de criterios y el método TOPSIS a fin de determinar el mejor orden de las alternativas. A pesar de que la toma de decisiones se puede realizar mediante FAHP, la integración con otras herramientas de decisión multicriterio mejora el proceso de decisión (Prakash y Barua, 2015). A continuación, se detallan los pasos a seguir.

El proceso de jerarquía analítica difuso o FAHP se utiliza para proporcionar la importancia de los criterios en la selección de localización. Los pasos son:

- Paso 1. Identificación de los criterios para la selección de la localización.

- Paso 2. Creación de la matriz difusa de comparación de criterios.

- Paso 3. Determinación de los pesos o ponderación de cada criterio según Büyüközkan, Kahraman y Ruan (2004).

TOPSIS se utiliza con la finalidad de realizar una clasificación o ranquin de las alternativas de localización en la que se utilizan los valores normalizados de los elementos en la matriz de decisión (Hwang y Yoon, 1981). Los pasos son:

- Paso 1. Elaborar una matriz de desempeño con el fin de evaluar cada alternativa de localización con respecto a cada uno de los criterios que se consideran.

- Paso 2. Construir la matriz ponderada normalizada multiplicando los elementos por

las ponderaciones de los criterios determinadas con AHP difuso.

- Paso 3. Determinar las soluciones ideal positiva e ideal negativa.

- Paso 4. Calcular la distancia de cada alternativa a la solución ideal positiva y de la solución ideal negativa.

- Paso 5. Calcular el coeficiente de proximidad o Ri.

- Paso 6. Ordenar las alternativas utilizando los valores de Ri. Cuanto mayor sea la puntuación mejor será la alternativa. 
Figura 1. Esquema metodológico propuesto

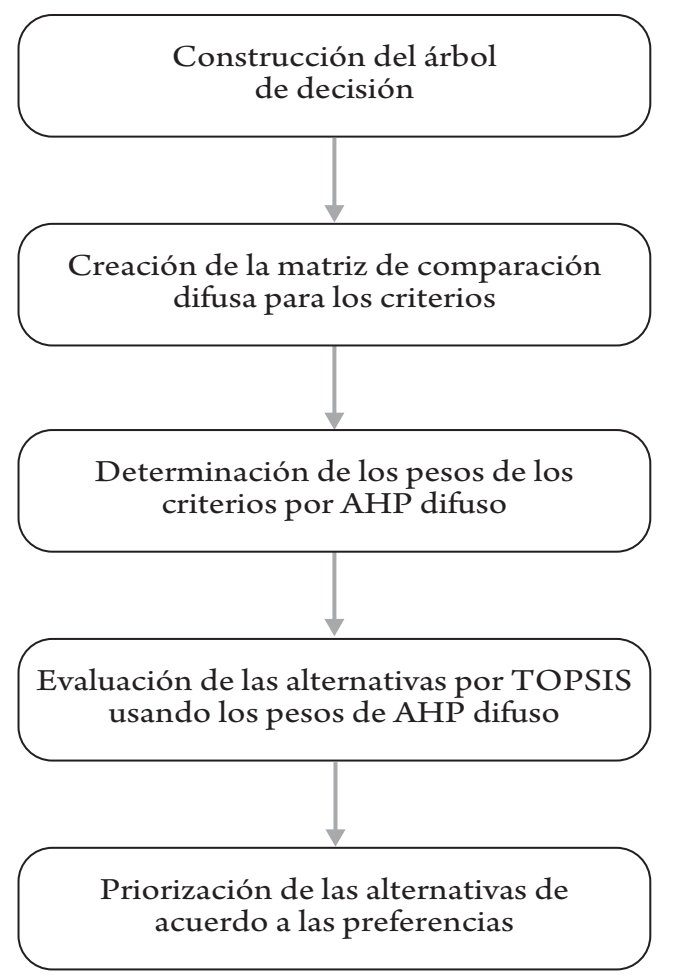

Fuente. Elaboración propia.

\section{Estudio de caso: aplicación del método propuesto para la selección de localización de locales comerciales}

$\mathrm{E}$ l caso de aplicación se desarrolla en Barranquilla, Colombia, con el propósito de identificar la mejor ubicación de una tienda de ropa deportiva en un centro comercial de la ciudad. Inicialmente, se conformó un grupo de expertos para la identificación de los centros comerciales y de los criterios de selección de localización.

Los centros comerciales que se han propuesto para ubicarlatienda son los siguientes: CCAmericano-A1, CC Buenavista-A2, CC Miramar-A3, CC Único-A4, CC Portal del Prado-A5 y CC Viva-A6. Los criterios de selección son: infraestructura del centro comercial-Crit.1, costo de arrendamiento-Crit.2, accesibilidad de clientes-Crit. 3 y potencial comercial-Crit.4.

\subsection{Determinación de los pesos de los criterios utilizando AHP difuso}

Una vez se determinan los criterios, el siguiente paso se dedica a obtener los pesos de prioridad de cada atributo, es decir, los criterios. Las comparaciones por pares se realizan en términos lingüísticos y sus números difusos triangulares, tal como se define en la 
tabla 1. Luego, se inserta la información requerida para el análisis y se obtiene la matriz difusa de comparación de los criterios principales con los pesos calculados que se presentan en la tabla 2.

Tabla 1. Escala de comparaciones por pares

\begin{tabular}{|c|c|c|}
\hline Escala lingüística & Escala numérica & Escala triangular difusa \\
\hline Igualmente importante & 1 & $(1,1,2)$ \\
\hline Moderadamente más importante & 3 & $(2,3,4)$ \\
\hline Fuertemente más importante & 5 & $(4,5,6)$ \\
\hline Absolutamente más importante & 7 & $(6,7,8)$ \\
\hline Muy fuertemente más importante & 9 & $(8,9,9)$ \\
\hline Valores intermedios & $2,4,6,8$ & $(1,2,3)(3,4,5)(5,6,7)(7,8,9)$ \\
\hline
\end{tabular}

Fuente. Guerrero, Marín y Gómez 2016.

Tabla 2. Matriz de comparaciones difusa

\begin{tabular}{|c|c|c|c|c|c|c|c|c|c|c|c|c|}
\hline & \multicolumn{4}{|c|}{ C1 } & \multicolumn{3}{c|}{ C2 } & \multicolumn{3}{c|}{ C3 } & \multicolumn{3}{c|}{ C4 } \\
\hline C1 & 1 & 1 & 1 & $1 / 3$ & $1 / 2$ & 1 & 2 & 3 & 4 & $1 / 5$ & $1 / 4$ & $1 / 3$ \\
\hline C2 & 1 & 2 & 3 & 1 & 1 & 1 & 1 & 2 & 3 & $1 / 3$ & $1 / 2$ & 1 \\
\hline C3 & $1 / 4$ & $1 / 3$ & $1 / 2$ & $1 / 3$ & $1 / 2$ & 1 & 1 & 1 & 1 & $1 / 6$ & $1 / 5$ & $1 / 4$ \\
\hline C4 & 3 & 4 & 5 & 1 & 2 & 3 & 4 & 5 & 6 & 1 & 1 & 1 \\
\hline
\end{tabular}

Fuente. Elaboración propia.

Se procede a determinar los pesos de cada criterio de acuerdo con lo que plantean Büyüközkan et al. (2004), se calcula inicialmente el grado sintético difuso (Tabla 3), posteriormente se comparan los números difusos de la matriz de criterios (Tabla 4) y, por último, se realiza la normalización a fin de obtener el vector de peso de los criterios de dicha matriz (Tabla 5).

Tabla 3. Grado sintético difuso

\begin{tabular}{|l|l|l|l|}
\hline $\mathrm{C} 1$ & 0,110 & 0,196 & 0,360 \\
\hline $\mathrm{C} 2$ & 0,104 & 0,226 & 0,454 \\
\hline $\mathrm{C} 3$ & 0,055 & 0,084 & 0,156 \\
\hline $\mathrm{C} 4$ & 0,281 & 0,494 & 0,851 \\
\hline
\end{tabular}

Fuente. Elaboración propia.

Tabla 4. Comparación de números difusos

\begin{tabular}{|c|c|c|c|c|c|c|c|}
\hline $\mathrm{C} 1-\mathrm{C} 2$ & 0,89 & $\mathrm{C} 2-\mathrm{C} 1$ & 1 & $\mathrm{C} 3-\mathrm{C} 1$ & 0,29 & $\mathrm{C} 4-\mathrm{C} 1$ & 1 \\
\hline $\mathrm{C} 1-\mathrm{C} 3$ & 1 & $\mathrm{C} 2-\mathrm{C} 3$ & 1 & $\mathrm{C} 3-\mathrm{C} 2$ & 0,27 & $\mathrm{C} 4-\mathrm{C} 2$ & 1 \\
\hline $\mathrm{C} 1-\mathrm{C} 4$ & 0,21 & $\mathrm{C} 2-\mathrm{C} 4$ & 0,39 & $\mathrm{C} 3-\mathrm{C} 4$ & 0 & $\mathrm{C} 4-\mathrm{C} 3$ & 1 \\
\hline
\end{tabular}

Fuente. Elaboración propia. 
Tabla 5. Peso normalizado de criterios

\begin{tabular}{|l|l|}
\hline $\mathrm{C} 1$ & 0,1119 \\
\hline $\mathrm{C} 2$ & 0,2103 \\
\hline $\mathrm{C} 3$ & 0,1432 \\
\hline $\mathrm{C} 4$ & 0,5346 \\
\hline
\end{tabular}

Fuente. Elaboración propia.

\subsection{Aplicación de TOPSIS en el ranquin de ubicaciones alternativas}

Se procede a la aplicación del método TOPSIS para la selección de la mejor localización. Los pesos de cada criterio calculados por FAHP se usan como valores de entrada para el TOPSIS. A fin de identificar cada alternativa y su respectiva evaluación, el grupo de expertos consideró diferentes factores que satisfacen todos los criterios (Tabla 6).

Tabla 6. Matriz de calificación de las alternativas

\begin{tabular}{|c|c|c|c|c|c|c|c|}
\hline & PESO & A1 & A2 & A3 & A4 & A5 & A6 \\
\hline C1 & 0,1119 & 4 & 7 & 2 & 5 & 4 & 8 \\
\hline C2 & 0,2103 & 8 & 4 & 7 & 9 & 6 & 3 \\
\hline C3 & 0,1432 & 3 & 8 & 2 & 4 & 5 & 6 \\
\hline C4 & 0,5346 & 6 & 7 & 5 & 7 & 6 & 8 \\
\hline
\end{tabular}

Fuente. Elaboración propia.

De acuerdo con Hanine, Boutkhoum, Tikniouine y Agouti (2016), el segundo paso en la técnica es la normalización de la matriz de calificaciones agregadas, tal como se ilustra en la tabla 7 . A continuación, se determinan las soluciones ideales positivas y negativas $-\mathrm{d}^{+}, \mathrm{d}-$, se calcula la distancia euclidiana de cada alternativa a las soluciones ideales y a partir de estos valores la proximidad relativa Ri; por último, se ordenan en sentido decreciente las alternativas (Tabla 8).

Tabla 7. Matriz de calificación normalizada

\begin{tabular}{|c|c|c|c|c|c|c|}
\cline { 2 - 7 } \multicolumn{1}{c|}{} & A1 & A2 & A3 & A4 & A5 & A6 \\
C1 & 0,0339 & 0,05936 & 0,017 & 0,0424 & 0,0339 & 0,0678 \\
\hline C2 & 0,1054 & 0,05269 & 0,0922 & 0,11854 & 0,079 & 0,0395 \\
\hline C3 & 0,0346 & 0,09229 & 0,0231 & 0,04615 & 0,0577 & 0,0692 \\
\hline C4 & 0,1993 & 0,23255 & 0,1661 & 0,23255 & 0,1993 & 0,2658 \\
\hline
\end{tabular}

Fuente. Elaboración propia.

Tabla 8. Proximidad relativa a la solución ideal con FAHP

\begin{tabular}{|c|c|c|c|c|}
\hline & $\mathrm{d}+$ & $\mathrm{d}-$ & $\mathrm{Ri}$ & Ranquin \\
\hline A1 & 0,078 & 0,038 & 0,3301 & 5 \\
\hline A2 & 0,042 & 0,081 & 0,6607 & 1 \\
\hline A3 & 0,088 & 0,071 & 0,4471 & 4 \\
\hline A4 & 0,087 & 0,035 & 0,2860 & 6 \\
\hline A5 & 0,043 & 0,050 & 0,5371 & 2 \\
\hline A6 & 0,079 & 0,082 & 0,5074 & 3 \\
\hline
\end{tabular}

Fuente. Elaboración propia. 


\section{Resultados y discusión}

$\mathrm{T}$ al como se muestra en la tabla 5, el criterio valorado con la mayor importancia es potencial comercial con una ponderación del $53,46 \%$, seguido por costos de arrendamiento con un 21,03\%; accesibilidad de clientes con un $14,32 \%$; y, finalmente, infraestructura física con el 11,19\%. Al observar las calificaciones de las alternativas en el criterio potencial comercial no hay mucha diferenciación, lo cual hace difícil la decisión; por tanto, la consideración de los otros criterios resultó definitiva para la selección final. Nótese que las alternativas A2 y A5 están por encima en el ordenamiento final sobre A6, que es la que tiene mejor calificación en el criterio de mayor ponderación.

Se realizó un análisis de sensibilidad con el fin de determinar el impacto de la apreciación dada por los tomadores de decisiones sobre la selección de la mejor ubicación para un almacén de ropa deportiva. En el análisis de sensibilidad se intercambian los pesos de dos criterios mientras que los otros dos permanecen constantes. Cada combinación se declara como un caso (Tabla 9).

Tabla 9. Análisis de sensibilidad

\begin{tabular}{|l|c|c|c|c|c|c|c|}
\hline & A1 & A2 & A3 & A4 & A5 & A6 & Ranquin \\
\hline Caso 1 - pesos orig.- & 0,3301 & 0,6607 & 0,4471 & 0,2860 & 0,5371 & 0,5074 & A2-A5-A6-A3-A1-A4 \\
\hline Caso 2 & 0,3796 & 0,5758 & 0,4884 & 0,2822 & 0,5775 & 0,4548 & A2-A5-A3-A6-A1-A5 \\
\hline Caso 3 & 0,3566 & 0,6022 & 0,4873 & 0,2782 & 0,5498 & 0,4893 & A2-A5-A6-A3-A1-A4 \\
\hline Caso 4 & 0,3639 & 0,4828 & 0,5180 & 0,2794 & 0,5599 & 0,4601 & A5-A3-A2-A6-A1-A4 \\
\hline Caso 5 & 0,2992 & 0,7180 & 0,3893 & 0,3030 & 0,5348 & 0,5145 & A2-A5-A6-A3-A4-A1 \\
\hline Caso 6 & 0,2097 & 0,8115 & 0,2275 & 0,2889 & 0,4017 & 0,7099 & A2-A6-A5-A4-A3-A1 \\
\hline Caso 7 & 0,1739 & 0,9067 & 0,1059 & 0,3276 & 0,4973 & 0,6568 & A2-A6-A5-A4-A1-A3 \\
\hline Caso 8 - pesos iguales- & 0,2297 & 0,6962 & 0,3227 & 0,3074 & 0,4963 & 0,5599 & A2-16-15-A3-A4-A1 \\
\hline
\end{tabular}

Fuente. Elaboración propia.

Adicionalmente, se expresó la matriz de comparación difusa de criterios como una matriz de números reales, lo cual se realizó para calcular los pesos de los criterios con la metodología AHP. A continuación, se muestran la matriz y las ponderaciones de cada criterio.

Tabla 10. Matriz de comparaciones de criterios con números reales

\begin{tabular}{|c|c|c|c|c|c|}
\hline & C1 & C2 & C3 & C4 & Wi \\
\hline $\mathbf{C 1}$ & 1 & $1 / 2$ & 3 & $1 / 4$ & 0,1656 \\
\hline $\mathbf{C 2}$ & 2 & 1 & 2 & $1 / 2$ & 0,2402 \\
\hline $\mathbf{C 3}$ & $1 / 3$ & $1 / 2$ & 1 & $1 / 5$ & 0,091 \\
\hline $\mathbf{C 4}$ & 4 & 2 & 5 & 1 & 0,5032 \\
\hline
\end{tabular}

Fuente. Elaboración propia. 
Estas nuevas ponderaciones se usaron como valores de entrada de TOPSIS.

Tabla 11. Proximidad relativa a la solución ideal con AHP

\begin{tabular}{|c|c|c|c|c|}
\hline & $\mathrm{d}+$ & $\mathrm{d}-$ & $\mathrm{Ri}$ & Ranquin \\
\hline $\mathrm{A} 1$ & 0,070 & 0,038 & 0,348 & 5 \\
\hline $\mathrm{A} 2$ & 0,049 & 0,068 & 0,581 & 1 \\
\hline $\mathrm{A} 3$ & 0,075 & 0,073 & 0,494 & 4 \\
\hline $\mathrm{A} 4$ & 0,087 & 0,033 & 0,277 & 6 \\
\hline $\mathrm{A} 5$ & 0,037 & 0,043 & 0,535 & 2 \\
\hline A6 & 0,083 & 0,083 & 0,500 & 3 \\
\hline
\end{tabular}

Fuente. Elaboración propia.

Aunque el nuevo ordenamiento es el mismo, se puede observar que con el método FAHP se logra una mejor discriminación entre las alternativas con respecto al ordenamiento con AHP, lo cual brinda al tomador de decisiones una mejor confianza en el proceso de selección.

\section{Conclusiones}

L a apertura de un nuevo local comercial es un factor crítico para las empresas del sector minorista, ya que la decisión conlleva una serie de riesgos financieros. Así, uno de los factores más importantes a tener en cuenta es su ubicación (Roig-Tierno, Baviera-Puig, Buitrago-Vera y Mas-Verdu, 2013). Por esta razón, el objetivo principal de este estudio es la selección de la localización con una combinación entre AHP difuso y el método TOPSIS. En este sentido, se presentó un estudio de aplicación en Barranquilla, Colombia, a fin de decidir la ubicación de una nueva tienda de ropa deportiva.

Durante un proceso de selección se dispone de un conjunto de alternativas que, además de cumplir con los requisitos, presentan características homogéneas. Esto dificulta el proceso de decisión, ya que las diferentes preferencias que tienen cada uno de los expertos en relación con cada criterio probablemente harán que la decisión sea más difícil de tomar. Aunque existen otros métodos y diferentes enfoques, el modelo AHP difuso-TOPSIS reduce la incertidumbre y mejora la precisión de la selección.
En la primera parte del estudio de caso se establecen las alternativas y los factores que influyen en la ubicación de una tienda de ropa deportiva. Se utilizó la metodología del proceso de jerarquía analítica difuso. En la segunda parte se elabora un ranquin de los centros comerciales mediante la metodología TOPSIS. Dado que los criterios y su clasificación relativa pueden variar de un tipo de negocio a otro, los resultados no pueden generalizarse. Estos criterios van a variar de acuerdo con el tipo de negocio que se va a ubicar en ellocal a seleccionar, pero la integración del FAHP y TOPSIS es una buena herramienta para incorporar la importancia de los criterios a tener en cuenta. Se realizó una comparación al ponderar los criterios mediante el proceso de jerarquía analítica. Se destaca que con la metodología desarrollada en este estudio se logra una mejor discriminación de los resultados, lo que brinda una mayor confianza en el proceso de selección.

Aunque los resultados finales pueden diferir, la metodología propuesta también se puede aplicar en otros sectores de negocios tales como el de restaurantes, sectores de servicios, centros de 
belleza, joyerías y almacenes de calzado, entre otros. El análisis de sensibilidad mostrado en el presente estudio muestra la solidez de la herramienta.

De esta forma, al utilizar el método de toma de decisiones del científico presentado en este documento, cualquier persona que tome decisiones encontrará una forma efectiva y confiable de seleccionar la mejor opción en un grupo de alternativas, y podrá cuantificar el valor subjetivo que tiene para cada opción según los diferentes criterios.

\section{Referencias}

Alizadeh, S.; Rad, M. M. S.; Bazzazi, A. A. (2016). Alunite processing method selection using the AHP and Topsis approaches under fuzzy environment. International Journal of Mining Science and Technology, 26(6), 1017-1023. DOI: https://doi.org/10.1016/j.ijmst.2016.09.009

Asakereh, A.; Soleymani, M.; Sheikhdavoodi, M. J. (2017). A GIS-based Fuzzy-AHP method for the evaluation of solar farms locations: case study in Khuzestan province, Iran. Solar Energy, 155, 342-353. DOI: https://doi.org/10.1016/j. solener.2017.05.075

Behzadian, M.; Otaghsara, S. K.; Yazdani, M.; Ignatius, J. (2012). A state-of the-art survey of Topsis applications. Expert Systems with Applications, 39(17), 13051-13069. DOI: https://doi.org/10.1016/j.eswa.2012.05.056

Beikkhakhian, Y.; Javanmardi, M.; Karbasian, M.; Khayambashi, B. (2015). The application of ISM model in evaluating agile supplier's selection criteria and ranking suppliers using fuzzy Topsis-AHP methods. Expert systems with Applications, 42(15-16), 6224-6236. DOI: https://doi.org/10.1016/j. eswa.2015.02.035

Bhushan, N.; Rai, K. (2007). Strategic decision making: applying the analytic hierarchy process. Springer Science \& Business Media.

Büyüközkan, G.; Kahraman, C.; Ruan, D. (2004). A fuzzy multi-criteria decision approach for software development strategy selection. International Journal of General Systems, 33(2-3), 259-280. DOI: https://doi.org/10.1080/030810 $\underline{70310001633581}$

Carlsson, C.; Fullér, R. (1996). Fuzzy multiple criteria decision making: recent developments. Fuzzy Sets and Systems, 78(2), 139-153. DOI: https://doi.org/10.1016/01650114(95)00165-4

Chauhan, A.; Singh, A. (2016). A hybrid multi-criteria decision making method approach for selecting a sustainable location of healthcare waste disposal facility. Journal of Cleaner Production, 139, 1001-1010. DOI: https://doi. org/10.1016/j.jclepro.2016.08.098
Chen, L. F.; Tsai, C. T. (2016). Data mining framework based on rough set theory to improve location selection decisions: a case study of a restaurant chain. Tourism Management, 53, 197-206. DOI: https://doi.org/10.1016/j. tourman.2015.10.001

Dožić, S.; Lutovac, T.; Kalić, M. (2018). Fuzzy AHP approach to passenger aircraft type selection. Journal of Air Transport Management, 68, 165-175. DOI: https://doi.org/10.1016/j. jairtraman.2017.08.003

Guerrero, L. C. U.; Marín, L. S. M.; Gómez, J. C. O. (2016) Selección multicriterio de aliado estratégico para la operación de carga terrestre. Estudios Gerenciales, 32(138), 35-43. DOI: https://doi.org/10.1016/i.estger.2015.09.002

Gürcan, Ö. F.; Beyca, Ö. F.; Arslan, Ç. Y.; Eldemir, F. (2016). Third party logistics (3PL) provider selection with AHP application. Procedia-Social and Behavioral Sciences, 235, 226-234. DOI: https://doi.org/10.1016/j. sbspro.2016.11.018

Hanine, M.; Boutkhoum, O.; Tikniouine, A.; Agouti, T. (2016). Application of an integrated multi-criteria decision making AHP-Topsis methodology for ETL software selection. SpringerPlus, 5(1), 263. DOI: https://doi.org/10.1186/ $\underline{\mathrm{s} 40064-016-1888-\mathrm{z}}$

Hualpa-Zúñiga, A. M.; Suárez-Roldán, C. (2013). Ubicación y dimensionamiento como parámetros en el diseño de almacenes: revisión del estado de arte. Ingeniería, 18(1), 65-83. DOI: https://doi.org/10.14483/udistrital.jour. reving.2013.1.a04

Hwang, C. L.; Yoon, K. (1981). Methods for multiple attribute decision making. En Multiple attribute decision making. (58-191). Berlin, Heidelberg: Springer. DOI: https://doi. org/10.1007/978-3-642-48318-9

Hong, L.; Xiaohua, Z. (2011). Study on location selection of multi-objective emergency logistics center based on AHP. Procedia Engineering, 15, 2128-2132. DOI: https://doi. org/10.1016/j.proeng.2011.08.398 
Hsieh, M. C.; Wang, E. M. Y.; Lee, W. C.; Li, L. W.; Hsieh, C. Y.; Tsai, W.; Wang, C. P.; Huang, J.L.; Liu, T. C. (2018). Application of HFACS, fuzzy TOPSIS, and AHP for identifying important human error factors in emergency departments in Taiwan. International Journal of Industrial Ergonomics, 67, 171-179. DOI: https://doi.org/10.1016/j. ergon.2018.05.004

Jayawickrama, H. M. M. M.; Kulatunga, A. K.; Mathavan, S. (2017). Fuzzy AHP based plant sustainability evaluation method. Procedia Manufacturing, 8, 571-578. DOI: https:// doi.org/10.1016/j.promfg.2017.02.073

Jumaah, F. M.; Zaidan, A. A.; Zaidan, B. B.; Bahbibi, R.; Qahtan, M. Y.; Sali, A. (2018). Technique for order performance by similarity to ideal solution for solving complex situations in multi-criteria optimization of the tracking channels of GPS baseband telecommunication receivers. Telecommunication Systems, 68(3), 425-443. DOI: https://doi.org/10.1007/ $\underline{\text { s11235-017-0401-5 }}$

Kazazi, S.; Akbari, A.; Jabbari, M.; Asefi, H. (2018). Parking lot site selection using a fuzzy AHP-Topsis framework in Tuyserkan, Iran. Journal of Urban Planning and Development, 144(3), 04018022. DOI: https://doi.org/10.1061/(ASCE) $\underline{\text { UP.1943-5444.0000456 }}$

Mosadeghi, R.; Warnken, J.; Tomlinson, R.; Mirfenderesk, H. (2015). Comparison of Fuzzy-AHP and AHP in a spatial multi-criteria decision making model for urban land-use planning. Computers, Environment and Urban Systems, 49, 54-65. DOI: https://doi.org/10.1016/j. compenvurbsys.2014.10.001

Prakash, C.; Barua, M. K. (2015). Integration of AHP-Topsis method for prioritizing the solutions of reverse logistics adoption to overcome its barriers under fuzzy environment. Journal of Manufacturing Systems, 37, 599-615. DOI: https:// doi.org/10.1016/j.jmsy.2015.03.001

Roig-Tierno, N.; Baviera-Puig, A.; Buitrago-Vera, J.; Mas-Verdu, F. (2013). The retail site location decision process using GIS and the analytical hierarchy process. Applied Geography, 40, 191-198. DOI: https://doi.org/10.1016/j. apgeog.2013.03.005

Saaty, T. L. (1980). The analytic hierarchy process. Nueva York: McGraw-Hill. DOI: https://doi.org/10.21236/ $\underline{\text { ADA214804 }}$
Saaty, T. L. (2008). Decision making with the analytic hierarchy process. International Journal of Services Sciences, 1(1), 83-98. DOI: https://doi.org/10.1504/IJSSCI.2008.017590

Santis, R.; Golliat, L.; Aguiar, E. (2017). Multi-criteria supplier selection using fuzzy analytic hierarchy process: case study from a Brazilian railway operator. Brazilian Journal of Operations \& Production Management, 14(3), 428-437. DOI: https://doi.org/10.14488/BJOPM.2017.v14.n3.a15

Sennaroglu, B.; Celebi, G. V. (2018). A military airport location selection by AHP integrated Promethee and Vikor methods. Transportation Research Part D: transport and Environment, 59, 160-173. DOI: https://doi.org/10.1016/j. trd.2017.12.022

Senouci, M. A.; Mushtaq, M. S.; Hoceini, S.; Mellouk, A. (2016). Topsis-based dynamic approach for mobile network interface selection. Computer Networks, 107, 304-314. DOI: https://doi.org/10.1016/j.comnet.2016.04.012

Sevtsuk,A. (2014).Location and agglomeration: the distribution of retail and food businesses in dense urban environments. Journal of Planning Education and Research, 34(4), 374-393. DOI: https://doi.org/10.1177/0739456X14550401

Walczak, D.; Rutkowska, A. (2017). Project rankings for participatory budget based on the fuzzy Topsis method. European Journal of Operational Research, 260(2), 706-714. DOI: https://doi.org/10.1016/j.ejor.2016.12.044

Yıldız, N.; Tüysüz, F. (2018). A hybrid multi-criteria decision making approach for strategic retail location investment: application to Turkish food retailing. Socio-Economic Planning Sciences, 68. DOI: https://doi.org/10.1016/j. seps.2018.02.006

Zhao, L.; Li, H.; Li, M.; Sun, Y.; Hu, Q.; Mao, S.; Li, J.; Xue, J. (2018). Location selection of intra-city distribution hubs in the metro-integrated logistics system. Tunnelling and Underground Space Technology, 80, 246-256. DOI: https:// doi.org/10.1016/j.tust.2018.06.024 
